\title{
Diversidad y estructura genética del Prochilodus magdalenae (Pisces: Prochilodontidae) aguas arriba y abajo de la represa Betania, Colombia
}

\section{Diversity and genetic structure of Prochilodus magdalenae (Pisces: Prochilodontidae) upstream and downstream Betania dam, Colombia}

\author{
Paulin P. Fontalvo@ ${ }^{1 *}$, Gilberto Orozco-Berdugo@² y Juan Narváez-Barandica $\bigodot^{3}$ \\ *Autor de correspondencia: paupol001@gmail.com
}

Recibido: 17 abril de 2018 - Aceptado: 11 de julio de 2018

1 Grupo de investigación de Biodiversidad y Ecología Aplicada, Universidad del Magdalena, Santa Marta, Colombia

2 CECIMAR, Universidad Nacional de Colombia, Sede Caribe, San Andrés y Providencia, Colombia

3 Grupo de investigación de Biodiversidad y Ecología Aplicada, Universidad del Magdalena, Santa Marta, Colombia

\begin{abstract}
Resumen
Palabras clave:

El río Magdalena es una de las cuencas más importantes de Colombia, cuenta con gran cantidad de

peces reofílicos;

microsatélites; fragmentación de ecosistemas; variabilidad

especies de peces importantes para la economía de muchas comunidades en el país. Sin embargo, una reducción marcada en las poblaciones de distintas especies atribuido a diferentes factores. Dentro de estos, se destaca la construcción de la represa de Betania, como respuesta a los requerimientos energéticos en Colombia. No obstante, especies migratorias como Prochilodus magdalenae, de importancia económica para la pesquería artesanal, se han visto seriamente afectadas, por lo que se ha generado la necesidad de analizar el hábitat y la diversidad genética, teniendo en cuenta la construcción de la represa como posible factor de fragmentación. Para esto, se hace adecuado el uso de microsatélites como marcador molecular en diferentes localidades de estudio aguas arriba y abajo de la represa. Esto permitió evaluar el estado de la diversidad genética en 171 organismos, cómo se encuentra distribuida esta variabilidad en las localidades de estudio y cómo influyen algunos factores como la construcción de la represa sobre su estructura genética. En este sentido, se obtuvieron 111 alelos distribuidos en siete loci $100 \%$ polimórficos. El promedio general para la heterocigosidad observada fue de 0,2169, mientras que para las esperadas fue de 0,8316. Los estadísticos de Fst y PhiPT mostraron que existe una moderada diferenciación entre la represa de Betania y las demás localidades muestreadas. El análisis de inferencia bayesiana detectó la coexistencia de tres poblaciones en la cuenca del río, entre las que destaca la de Betania. Estos resultados se pueden estar presentando, debido a la fragmentación que genera la represa en el río, impidiendo el flujo genético entre las localidades aguas arriba y aguas abajo de la represa.
\end{abstract}

Key words:

rheophilics fish; microsatellites; ecosystem fragmentation; genetic variability

\begin{abstract}
The Magdalena River is one of the most important basins in Colombia, it has a large number of important fish species for the economy of many communities in the country. However, there's been a reduction in the population caused by different factors. One of the major problems is the construction of the Betania dam, in response to the energy requirements in Colombia. However, migratory species such as Prochilodus magdalenae, of economic importance for the artisanal fishery, have been seriously affected. For this reason, it became neccesary to analyze its genetic diversity and habitat, taking into account the construction of the dam as a possible factor of fragmentation. For this purpose, the use of microsatellites as a molecular marker in different study locations upstream and downstream of the dam is adequate. It was possible to evaluate in 171 individuals the state of genetic diversity. In this respect, 111 alleles distributed in seven loci $100 \%$ polymorphic were obtained. The overall averages for the heterozygosity observed were 0.2169 , while the expected ones were 0.8316 . The Fst and PhiPT statistics showed that there is a moderate differentiation between Betania's locality and all the localities sampled. The analysis of Bayesian inference detected the coexistence of three populations in the river basin, being the Betania's locality represented only by one of the three populations registered in the basin. These results can be presented due to the fragmentation generated by the dam in the river, preventing the genetic flow between the locations upstream and downstream of the dam.
\end{abstract}




\section{Introducción}

La fragmentación es considerada como uno de los principales factores responsables de la alteración del medio ambiente y de los ecosistemas; ya que afecta la función, la estructura y la composición en determinado espacio natural (Instituto Alexander von Humboldt, 2002). Estas afectaciones provocan una disminución en la conectividad y/o crea bordes en el hábitat, lo que genera cambios en el comportamiento de las especies asociadas a los ambientes fragmentados y, por ende, aumenta la vulnerabilidad de los organismos (Bustamante y Grez, 1995). Pese a los reconocidos efectos de la fragmentación del hábitat en la dinámica ecosistémica, la construcción de barreras artificiales, como las represas, está ligadas al desarrollo económico y social de una comunidad, para dar soluciones al requerimiento de recursos como agua y energía a las poblaciones humanas (Agostinho et al., 2008; JiménezSegura et al., 2014). Sin embargo, su construcción ha llegado a generar desde reducciones o aumentos en el número de especies de peces (Agostinho et al., 2001) hasta extinciones locales asociadas a la cuenca intervenida (Rodríguez, 2015).

Cabe señalar que la fragmentación impide el flujo de las especies migratorias en los cauces de los ríos (Pompeu et al., 2009). Por lo anterior, la construcción descomedida de represas en Sudamérica ha ido deteriorando seriamente los sistemas lóticos, especialmente el componente biótico (Kopas y Puentes, 2009). Es así como se han presentado casos en que algunas especies de peces han incrementado 0 , en caso contrario, han disminuido a causa de la construcción de la represa (Agostinho et al., 2008). Esta problemática ha despertado el interés de la comunidad científica internacional para entender cómo responden las poblaciones de especies reofílicas frente a la construcción de estas barreras físicas a nivel molecular. Es así como se hace necesaria la implementación y uso de herramientas genéticas, para analizar la pérdida de variabilidad en las poblaciones afectadas. Por lo anterior, el uso de microsatélites ha resultado muy útil para analizar aspectos genéticos en términos globales de muchas especies de peces migratorios, como el género Prochilodus, un modelo biológico adecuado para entender cómo responden genéticamente estos organismos frente a las diferentes barreras naturales y artificiales que los pueden afectar (Carvalho-Costa et al., 2008; Jiménez-Segura et al., 2010; Silva, 2011; Barroca et al., 2012a; Orozco y Narváez, 2014; Pelecice et al., 2014; Guevara, 2015).

El río Magdalena, considerado como la principal cuenca hidrográfica del país, es uno de los medios de apoyo económico más importantes, por ejemplo, para muchas comunidades cuyo sustento se basa en la pesca (JiménezSegura et al., 2010). Alberga alrededor de 167 especies de peces, de las cuales se puede resaltar a Prochilodus magdalenae (Steindachner, 1879); especie endémica, conocida comúnmente como bocachico (MaldonadoOcampo et al., 2005), la cual es aprovechada por la pesquería artesanal en la cuenca. Esta hace parte de la familia de peces Prochilodontidae y se caracteriza por presentar una espina predorsal muy afilada, boca pequeña, prominente y pequeños dientes, tal como se observa en la figura 1 (Mojica et al., 2012). El bocachico como especie migratoria tiene dos picos reproductivos anuales que concuerdan con los patrones hidrológicos del río Magdalena. En este sentido, el primer pico reproductivo inicia entre diciembre y enero, mientras que el segundo, entre julio y septiembre. En este último se cree que sólo se reproducen aquellos individuos que no pudieron hacerlo durante el primer ciclo hidrológico del río (Jiménez-Segura et al., 2010).

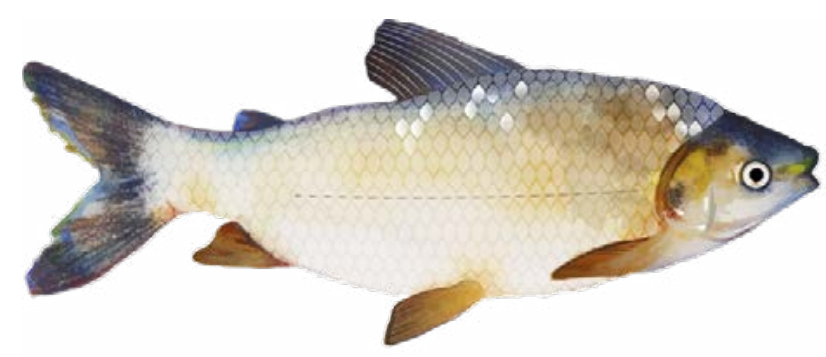

Figura 1. Ilustración de Prochilodus magdalenae (Ilustración digital de María José Cárdenas Angulo).

Debido a su importancia pesquera, esta especie ha sido sometida a estudios ecológicos (Jiménez-Segura et al., 2010; Ramírez y Pinilla, 2012), reproductivos (Atencio, 2001; Cortés, 2003) y de conservación (Cortés, 2003; Maldonado-Ocampo et al., 2005), pero pocos han sido los trabajos a nivel molecular en Colombia. No obstante, entre los estudios desarrollados con microsatélites, se pueden destacar los realizados para la especie Prochilodus magdalenae (Santacruz-Beltrán, 2003; Orozco y Narváez, 2014; Guevara, 2015) (ver tabla 5), donde se exhiben para todos los casos bajos valores de variabilidad debido a diferentes factores que incluyen la sobrepesca, barreras tanto artificiales como naturales, entre otras, lo que permite evidenciar el estado crítico de la especie (Orozco y Narváez, 2014).

Por lo tanto, considerando la distribución geográfica de esta especie (Maldonado-Ocampo et al., 2005) y su categoría 
de amenaza como vulnerable (Mojica et al., 2012), el uso de microsatélites como marcadores moleculares resulta útil para evaluar la estructura y diversidad genética del bocachico posterior a la construcción de la represa de Betania en 1987, en la parte media y alta de la cuenca del río Magdalena.

\section{Materiales y métodos}

\section{Área de estudio}

La cuenca del río Magdalena ocupa un área de 257438 $\mathrm{km}^{2}$, representando un $24 \%$ del territorio nacional que, además, colinda con 18 departamentos donde cerca del $80 \%$ de la población depende de esta cuenca por ser un importante eje de desarrollo económico (Lasso et al., 2011). Presenta tres divisiones que son: Alto Magdalena, con una altura aproximada desde su nacimiento en los 3685 hasta Honda en los 229 m.s.n.m. La segunda división, llamada Medio Magdalena, va desde los 229 hasta los 33 m.s.n.m. en el municipio de El Banco departamento del Magdalena. Finalizando, la tercera división o bajo Magdalena comprende la desembocadura del río Magdalena que va desde los 33 m.s.n.m. hasta el nivel del mar (IDEAM y Cormagdalena, 2001).

\section{Fase de campo}

Las muestras se obtuvieron con la ayuda de la flota artesanal y desembarques pesqueros en las cinco localidades descritas en la figura 2 . Se tuvo en cuenta el cuidado de la toma de muestras de tejido muscular junto a la flota artesanal, ya que dichas embarcaciones no tienen mucha autonomía y siempre pescan cerca del sitio de desembarque, lo que garantizó que los individuos capturados pertenecieran a las localidades seleccionadas. Debido a que la especie analizada en el presente estudio es muy comercial, su reconocimiento en campo no requirió de claves taxonómicas especializadas. No obstante, se tuvo en cuenta que efectivamente los individuos tuvieran las características morfológicas tales como: la espina predorsal punzante, 10 radios en la aleta dorsal, 11 radios en la aleta anal y entre 40 y 46 escamas perforadas que caracterizan la línea lateral (Mojica et al., 2012).

Un total de 171 especímenes se recolectaron en el cauce principal del río, en cuatro sitios ubicados aguas abajo de la represa de Betania en el río Magdalena y en tributarios como el río Saldaña, así como un sitio aguas arriba de la represa, entre los meses de abril del 2010 y noviembre del 2012 (figura 2). 40 individuos pertenecientes a la localidad de Boyacá, otros 40 de La Dorada, 19 de Neiva, 29 de
Betania y finalmente 43 de Saldaña. El tamaño muestral del presente estudio dependió del número de especímenes que fue recolectado por los pescadores en cada localidad durante el periodo de muestreo. Posterior a esto, se tomaron muestras de tejido muscular del pedúnculo caudal y se fijó en etanol al $96 \%$ para su conservación.

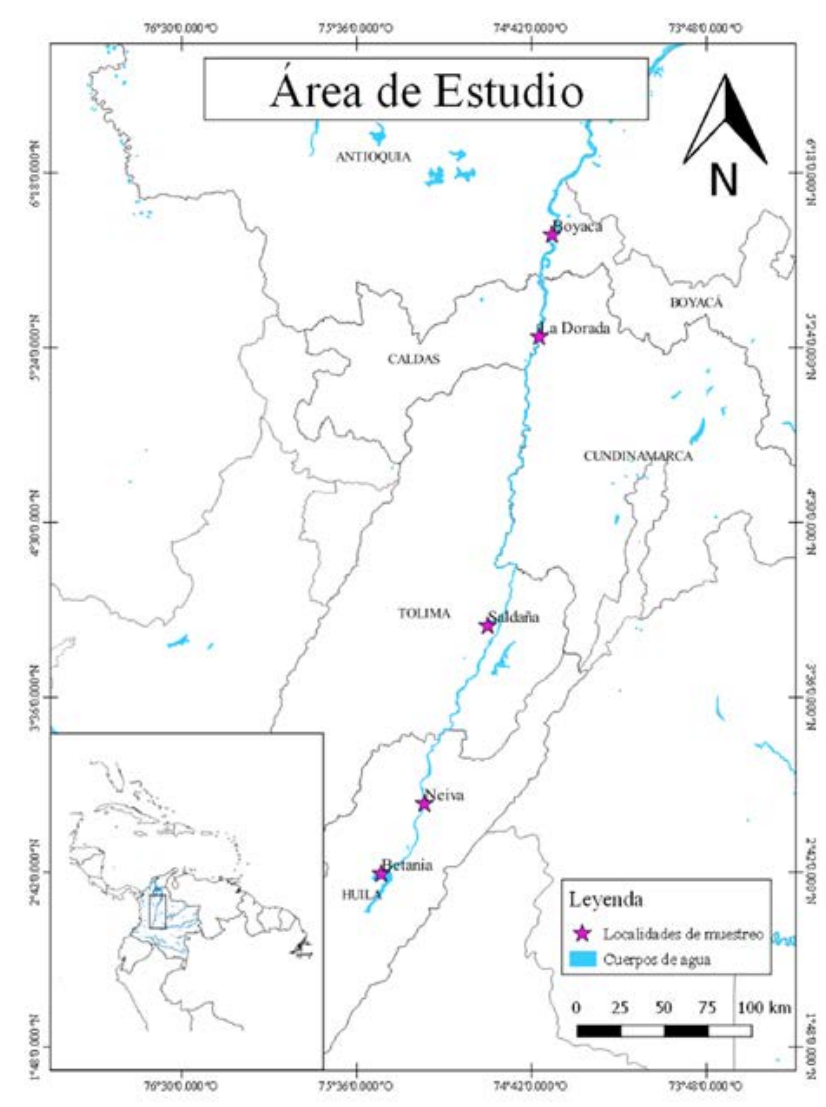

Figura 2. Mapa de las cinco localidades de estudio en la cuenca del río Magdalena, Colombia.

\section{Fase de laboratorio}

El ADN genómico se extrajo del músculo de la aleta caudal de los ejemplares recolectados, usando el kit MasterPureTM (Epicentre Biotechnologies ${ }^{\circledR}$. El ADN se verificó a través de un gel de agarosa al 0,8 \% teñido con GelRed y una corrida a 80 voltios durante 30 minutos. Se utilizaron siete loci de microsatélites (tabla 1), descritos para P. lineatus con una amplificación cruzada en $P$. magdalenae (Rueda et al., 2011). La reacción de amplificación se realizó siguiendo las condiciones propuestas por Rueda et al. (2011) y Orozco y Narváez (2014). El volumen final utilizado fue de $10 \mu \mathrm{L}$, conteniendo 100 ng de ADN, 1 X de reacción buffer, 2 mM 
de MgCl2, $200 \mu \mathrm{M}$ de dNTPs, $0,2 \mu \mathrm{M}$ de cada cebador y 0,25 $\mathrm{U}$ de Taq polimerasa. Las condiciones para la PCR fueron las siguientes: 5 minutos a $95^{\circ} \mathrm{C}, 30$ ciclos de $30 \mathrm{~s}$ a $94{ }^{\circ} \mathrm{C}$, $30 \mathrm{~s}$ para la temperatura de alineamiento (tabla 1), $30 \mathrm{~s} \mathrm{a}$ $72{ }^{\circ} \mathrm{C}$ y una temperatura de extensión final de $72{ }^{\circ} \mathrm{C}$ por 10 minutos. Las reacciones se analizaron en un termociclador con gradiente de temperatura ESCO-SWIFT MaxPro. Los productos de la amplificación fueron analizados por electroforesis capilar QIAxcel Advanced (QIAGEN) usando un kit de alta resolución (High Resolution Kit QIAGEN) y una escalera de peso molecular de concentración conocida (DNA SizeMarker 50-800 bp v2.0 QIAGEN).

El tamaño de cada producto amplificado se determinó con el programa ScreenGel QIaxcel v1.0 QIAGEN, el cual permitió cuantificar el peso de cada banda y, consecuentemente, distinguir el genotipo de cada individuo.

\section{Análisis de datos}

A partir de la matriz final de datos que contiene el genotipo de todos los individuos y con la ayuda del programa MSTOOLS, fue verificada la diferencia entre los tamaños de los alelos presentes en la población (Park, 2001). Este mismo programa se utilizó para la generación de los diferentes archivos de entrada utilizados en otros programas. Por ejemplo, la presencia de errores en la genotipificación derivados de artefactos técnicos, o pérdida de alelos por la mala calidad del ADN fue evaluada con el programa MICRO-CHECKER (Van Oosterhout et al., 2004), remuestreando los alelos de cada locus a través de 1000 simulaciones bootstrap; de esta manera, crearon intervalos de confianza entre la frecuencia esperada de homocigotos y heterocigotos para los alelos registrados.

Tabla 1. Descripción de cada microsatélite utilizado. Motivo de repetición, Temperatura ${ }^{\circ} \mathrm{C}, \mathrm{N}_{\mathrm{A}}$; Número de alelos, Tamaño en pares de bases. Tomado y modificado de Rueda et al. (2011).

\begin{tabular}{|c|c|c|c|c|c|}
\hline Locus & $\begin{array}{l}\text { Motivo de } \\
\text { repetición }\end{array}$ & $\mathrm{T}\left({ }^{\circ} \mathrm{C}\right)$ & $\mathbf{N}_{\mathrm{A}}$ & $\begin{array}{r}\text { Tamaño en } \\
\text { pares de bases }\end{array}$ & Secuencia del primer \\
\hline PL3 & $(\mathrm{CA}) \mathrm{n}$ & 50 & 6 & $185-203$ & $\begin{array}{l}\text { F: 5'-TCTGAGCTGTGAGGAATGGA-3' } \\
\text { R: } \quad \text { ''-AGAGCGCTCAAGCACAAGAT-3' }\end{array}$ \\
\hline PL14 & $(\mathrm{CA}) \mathrm{n}$ & 61 & 11 & $104-134$ & $\begin{array}{l}\text { F: 5'-TGCCCAACACTGAAACTGAG-3' } \\
\text { R: } \quad \text { '’'CTCATCAACCTGCCTGGAAT-3' }\end{array}$ \\
\hline PL23 & $(\mathrm{CA}) \mathrm{n}$ & 59 & 3 & $244-252$ & $\begin{array}{l}\text { F: 5'-TTGGCTACTTCCCCAAACAC-3' } \\
\text { R: } \quad \text { 5'-GGGGAACTAGTTTGACGATGC-3' }\end{array}$ \\
\hline PL28 & $(\mathrm{CA}) \mathrm{n}$ & 59 & 3 & $239-249$ & $\begin{array}{l}\text { F: 5'-GAAGCTTGGGCTCTTGACAT-3' } \\
\text { R: } \quad \text { ''-CGTTTGCCTCTAGCCTTTTG-3' }\end{array}$ \\
\hline PL34 & $(\mathrm{CA}) \mathrm{n}$ & 56 & 10 & $178-212$ & $\begin{array}{l}\text { F: 5'-GAGCGGATTCTCCACATGAT-3' } \\
\text { R: } \quad \text { 5'-TAATGTGCTCCCTCCCACAG-3' }\end{array}$ \\
\hline PL64 & $(\mathrm{CA}) \mathrm{n}$ & 62 & 13 & $158-180$ & $\begin{array}{l}\text { F: 5'-AGAGCAACACAGGGAGGAGT-3' } \\
\text { R: } \quad \text { ''-ACGCTCTGCTCAGCCATACT-3' }\end{array}$ \\
\hline PL119 & $(\mathrm{CA}) \mathrm{n}$ & 58 & 12 & $161-207$ & $\begin{array}{ll}\text { F: } & \text { 5'-GAAAAAGGCTAGGGGACTGG-3' } \\
\text { R: } & \text { 5'-GAGGAAAATTGCCTTTTGTAGG-3' }\end{array}$ \\
\hline
\end{tabular}

La diversidad genética fue determinada para cada población analizando el número de alelos por locus $\left(\mathrm{N}_{\mathrm{A}}\right)$, heterocigosidad observada (Ho) y heterocigosidad 90 esperada (He), usando el programa GenAlex 6.5 (Peakall y Smouse, 2006). El coeficiente de endogamia (Fis) para los siete loci se calcularon en FSTAT (Goudet, 1995). El equilibrio de Hardy-Weinberg $(\mathrm{p}<0,05)$ se estimó por medio de una prueba análoga a la prueba exacta de Fisher 
(Guo y Thompson, 1992) que tiene como hipótesis nula la unión aleatoria de gametos. Esta prueba fue estimada con una serie de permutaciones de la Cadena de Markov Monte Carlo (MCMC) (10 000 batches / 1000 iteraciones), implementados en GENEPOP (Raymond y Rousset, 1995).

La segregación independiente de los genotipos o desequilibrio de ligamiento, se comprobó usando pruebas exactas con MCMC (10 000 batches/ 1000 iteraciones) (Guo y Thompson, 1992), implementados en GENEPOP. Para verificar la distribución de la variancia molecular entre los grupos naturales de poblaciones, la existencia de poblaciones genéticamente diferenciadas de $P$. magdalenae entre las localidades del canal principal del río Magdalena y la represa; y para evaluar el grado de significancia de la variabilidad genética entre y dentro de las localidades, se realizó un Análisis de Varianza Molecular (AMOVA) en el programa ARLEQUÍN 3.1 (Schneider et al., 2000). Este análisis examinó la distribución de la variabilidad genética dentro de diferentes niveles jerárquicos a diferentes escalas geográficas en la cuenca del río Magdalena. En este caso, los niveles jerárquicos fueron evaluados así: 1 , todas las poblaciones como un grupo; 2 , un grupo compuesto por las localidades presentes en el cauce principal en el río Magdalena sin los tributarios (río Saldaña) y 3, finalmente considerando a la represa de Betania como un grupo independiente de las demás localidades. Además de esto, el programa ARLEQUIN 3.1 se empleó para calcular los valores de Fst por parejas (Weir y Cockerham, 1984) estimados para cada par poblacional. Fue utilizado el Fst, ya que este es el índice más usado para la estimación de distancias génicas en poblaciones de peces (O'connell y Wright, 1997), además de ser el más adecuado en estudios que contengan muestras de tamaño moderado a pequeño (menos de 50) y que utilicen menos de diez loci. Adicionalmente, se utilizó el índice PhiPT como prueba análoga, basado en la hipótesis que los loci no se encuentran bajo los supuestos de equilibrio de HardyWeinberg.

La metodología de agrupamiento bayesiano de STRUCTURE versión 2.3.3 (Hubisz et al., 2009) se aplicó para identificar grupos de individuos genéticamente similares y determinar el nivel de estructura genética en los datos independientes de las áreas muestreadas. Este programa realiza una prueba de asignación donde los individuos son clasificados según las probabilidades de pertenecer a uno o más grupos cuando la población es genéticamente mezclada.

Para estimar el número de subpoblaciones (K), 20 corridas independientes de $\mathrm{K}=1-8$ fueron llevadas a cabo con 10 000 repeticiones de MCMC, con 1000 periodos de burn-in, usando información LOC-PRIOR y asumiendo frecuencias alélicas correlacionadas (Allele Frequencies Correlated). El patrón de migración de $P$. magdalenae incluye la existencia de un fuerte flujo génico entre las poblaciones, por lo tanto, se usó el modelo de mezcla (Admixture Model) que supone que cada individuo tiene la misma proporción de herencia de su ancestro en cada población. Para determinar el número de poblaciones (K) presentes, se utilizó un método propuesto por Evanno et al. (2005). Este valor fue obtenido usando el programa STRUCTURE HARVESTER 0.56.3 (Earl y von Holdt, 2012).

\section{Resultados}

\section{Diversidad genética}

Todos los microsatélites evaluados fueron polimórficos (100 $\%$ ) en los ejemplares de $P$. magdalenae en las diferentes localidades del río Magdalena. Un total de 111 alelos se encontraron distribuidos en los cinco sitios de muestreo. Betania, con un promedio de 8,7 de alelos por locus, fue la de menor cantidad, mientras que Saldaña, con 10,25, fue la de mayor promedio como se observa en la tabla 2. Los loci PL28 y PL34 presentaron menor cantidad de alelos (cinco cada uno), mientras que P119 tuvo 16 (tabla 2). Por otro lado, la Ho en promedio para todas las localidades fue de 0,21, la población de Saldaña presentó el índice más bajo con 0,11 y Betania fue la de mayor Ho con 0,27.

No obstante, la He fue considerablemente más alta que la Ho, con promedios para todas las localidades de 0,83 . Se obtuvieron valores entre 0,80 y 0,84 para los sitios de muestreo de Betania y Saldaña respectivamente, como se observa en la tabla 2. Todas las localidades muestreadas presentaron desequilibrio de Hardy-Weinberg asociado a un déficit de heterocigotos. Adicionalmente, se encontró que todos los loci de microsatélites utilizados en el presente estudio presentaron alelos nulos en los ejemplares analizados y no hubo desequilibrio de ligamiento.

\section{Estructura genética y diferenciación poblacional}

La prueba de Fst mostró un valor global de 0,065 e indicó una moderada diferenciación entre las localidades examinadas, según la escala de Wright (1978). Entre cada par de poblaciones, este índice presentó fluctuaciones que van desde localidades con baja diferenciación como Boyacá - La Dorada (Fst de 0,007), hasta localidades con moderada diferenciación como La Dorada y Saldaña (Fst de 0,094), tal como se observa en la tabla 3 diagonal inferior. 
Al igual que los valores de Fst, el índice de PhiPT mostró valores que van desde una baja a una moderada diferenciación genética entre la mayoría de las localidades. Es importante resaltar que, a pesar de la poca distancia geográfica entre Neiva y la represa de Betania, los valores registrados para estos índices evidenciaron una moderada diferenciación genética entre localidades.

Debido a que todas las localidades presentaron desviaciones en el equilibrio de Hardy-Weinberg, se realizó la prueba no paramétrica análoga a Fst: PhiPT para estimar la diferenciación genética entre las localidades. La prueba mostró que entre las localidades de Boyacá - La Dorada y Neiva - La Dorada, se registraron los valores más bajos para este índice, lo que indica una baja diferenciación entre estas localidades. En los demás análisis pareados entre localidades, se encontró una moderada diferenciación genética, como se observa en la tabla 3. Cabe resaltar que la represa de Betania registró los valores más altos para este índice.

Tabla 2. Tabla general de resultados. $\mathrm{N}$; tamaño de la muestra, $\mathrm{N}_{\mathrm{A}}$; número de alelos, Ho; heterocigosidad observada, He; heterocigosidad esperada, F; índice de fijación y P; equilibrio de Hardy-Weinberg.

\begin{tabular}{|c|c|c|c|c|c|c|c|c|c|}
\hline \multirow{2}{*}{ Sitio } & \multirow{2}{*}{ Estadístico } & \multicolumn{7}{|c|}{ Loci } & \multirow{2}{*}{$\overline{\mathbf{x}}$} \\
\hline & & PL28 & PL14 & PL23 & PL34 & PL64 & PL119 & PL3 & \\
\hline \multirow{5}{*}{$\begin{array}{l}\text { Boyacá } \\
(\mathrm{N}=40)\end{array}$} & $\mathrm{N}_{\mathrm{A}}$ & 8 & 14 & 8 & 8 & 9 & 14 & 10 & 10,14 \\
\hline & Ho & 0,000 & 0,200 & 0,237 & 0,050 & 0,579 & 0,065 & 0,250 & 0,199 \\
\hline & $\mathrm{He}$ & 0,761 & 0,903 & 0,817 & 0,755 & 0,838 & 0,913 & 0,810 & 0,844 \\
\hline & $\mathrm{F}$ & 1,000 & 0,778 & 0,710 & 0,934 & 0,309 & 0,929 & 0,691 & 0,765 \\
\hline & $\mathrm{P}$ & $<0,001$ & $<0,001$ & $<0,001$ & $<0,001$ & $<0,001$ & $<0,001$ & $<0,001$ & $<0,001$ \\
\hline \multirow{5}{*}{$\begin{array}{l}\text { Dorada } \\
(\mathrm{N}=40)\end{array}$} & $\mathrm{N}_{\mathrm{A}}$ & 10 & 12 & 8 & 5 & 10 & 13 & 8 & 9,429 \\
\hline & Но & 0,050 & 0,310 & 0,175 & 0,075 & 0,475 & 0,406 & 0,308 & 0,25 \\
\hline & $\mathrm{He}$ & 0,800 & 0,878 & 0,818 & 0,684 & 0,830 & 0,891 & 0,778 & 0,825 \\
\hline & $\mathrm{F}$ & 0,938 & 0,647 & 0,786 & 0,890 & 0,428 & 0,544 & 0,605 & 0,691 \\
\hline & $\mathrm{P}$ & $<0,001$ & $<0,001$ & $<0,001$ & $<0,001$ & $<0,001$ & $<0,001$ & $<0,001$ & $<0,001$ \\
\hline \multirow{5}{*}{$\begin{array}{l}\text { Neiva } \\
(\mathrm{N}=19)\end{array}$} & $\mathrm{N}_{\mathrm{A}}$ & 7 & 13 & 9 & 6 & 8 & 16 & 6 & 9,286 \\
\hline & Ho & 0,000 & 0,211 & 0,316 & 0,176 & 0,500 & 0,211 & 0,368 & 0,254 \\
\hline & $\mathrm{He}$ & 0,715 & 0,891 & 0,861 & 0,607 & 0,792 & 0,925 & 0,785 & 0,838 \\
\hline & $\mathrm{F}$ & 1,000 & 0,764 & 0,633 & 0,709 & 0,368 & 0,772 & 0,531 & 0,683 \\
\hline & $\mathrm{P}$ & $<0,001$ & $<0,001$ & $<0,001$ & $<0,001$ & $<0,001$ & $<0,001$ & $<0,001$ & $<0,001$ \\
\hline \multirow{5}{*}{$\begin{array}{l}\text { Betania } \\
(\mathrm{N}=29)\end{array}$} & $\mathrm{N}_{\mathrm{A}}$ & 5 & 12 & 12 & 8 & 6 & 12 & 6 & 8,714 \\
\hline & Ho & 0,000 & 0,143 & 0,643 & 0,333 & 0,208 & 0,536 & 0,000 & 0,271 \\
\hline & $\mathrm{He}$ & 0,571 & 0,879 & 0,865 & 0,785 & 0,747 & 0,878 & 0,717 & 0,804 \\
\hline & $\mathrm{F}$ & 1,000 & 0,837 & 0,257 & 0,575 & 0,721 & 0,390 & 1,000 & 0,683 \\
\hline & $\mathrm{P}$ & $<0,001$ & $<0,001$ & $<0,001$ & $<0,001$ & $<0,001$ & $<0,001$ & $<0,001$ & $<0,001$ \\
\hline \multirow{5}{*}{$\begin{array}{l}\text { Saldaña } \\
(\mathrm{N}=43)\end{array}$} & $\mathrm{N}_{\mathrm{A}}$ & 6 & 13 & 10 & 9 & 9 & 16 & 9 & 10,29 \\
\hline & Ho & 0,000 & 0,122 & 0,116 & 0,023 & 0,140 & 0,186 & 0,186 & 0,11 \\
\hline & $\mathrm{He}$ & 0,812 & 0,888 & 0,777 & 0,785 & 0,794 & 0,913 & 0,832 & 0,847 \\
\hline & $\mathrm{F}$ & 1,000 & 0,863 & 0,850 & 0,970 & 0,824 & 0,796 & 0,776 & 0,869 \\
\hline & $\mathrm{P}$ & $<0,001$ & $<0,001$ & $<0,001$ & $<0,001$ & $<0,001$ & $<0,001$ & $<0,001$ & $<0,001$ \\
\hline
\end{tabular}


El AMOVA, basado en la agrupación de las localidades en diferentes niveles o escalas jerárquicas, mostró que al tomar a todas las localidades muestreadas como un solo grupo, se registró un valor Fst de 0,065 que indica una moderada diferenciación según la escala de Wright (1978). Aproximadamente el 6,6 \% de la diversidad detectada por los microsatélites está distribuida entre las poblaciones, mientras que cerca de 93,41\% está distribuido dentro de las poblaciones, como se muestra en la tabla 4.

El segundo agrupamiento tuvo como objetivo evaluar el efecto de los tributarios del río Magdalena sobre la distribución de la variación genética de la población. En este segundo grupo, se eliminó al río Saldaña del análisis, y se encontró un valor de Fst de 0,031 indicó la inexistencia de diferenciación genética entre las localidades (tabla 4). El tercer agrupamiento tuvo por objetivo evaluar si la construcción de la barrera había causado un efecto sobre el acervo genético de la población. Lo anterior mostró un valor bajo en el índice de Fst $(0,017)$ Registrado en la tabla 4.

El AMOVA y los índices Fst y PhiPT, requieren de una definición a priori de las subpoblaciones o localidades muestreadas, y ese agrupamiento puede no corresponder a verdaderos grupos biológicos. Por lo tanto, para verificar la veracidad de la diferenciación encontrada, se realizó un análisis bayesiano en el programa STRUCTURE, el cual identificó la presencia de tres poblaciones que coexisten en la cuenca del río Magdalena, como se observa en la figura 3.

Tabla 3. Diagonal inferior: estimación del índice de Fst entre pares de localidades de P. magdalenae de las cinco localidades muestreadas, usando siete loci de microsatélites. Diagonal superior; Estimación del índice de PhiPT entre pares de individuos de P. magdalenae de los cinco sitios muestreados, usando siete loci de microsatélites.

\begin{tabular}{lrrrrr}
\hline Población & Boyacá & Dorada & Neiva & Betania & Saldaña \\
\hline Boyacá & 0 & 0,010 & 0,051 & 0,056 & 0,054 \\
Dorada & 0,007 & 0 & 0,047 & 0,057 & 0,053 \\
Neiva & 0,074 & 0,072 & 0 & 0,059 & 0,054 \\
Betania & 0,085 & 0,091 & 0,078 & 0 & 0,061 \\
Saldaña & 0,093 & 0,094 & 0,078 & 0,091 & 0 \\
\hline
\end{tabular}

Tabla 4. Análisis molecular de varianza (AMOVA) en las localidades de P. magdalenae.

\begin{tabular}{|c|c|c|c|c|c|c|}
\hline AMOVA & Muestras & $\begin{array}{r}\text { \% Entre } \\
\text { grupos }\end{array}$ & $\begin{array}{r}\text { \% Dentro de } \\
\text { los grupos }\end{array}$ & $\begin{array}{r}\% \text { de } \\
\text { variación }\end{array}$ & p-valor & Fst \\
\hline \multirow{3}{*}{ Por niveles } & $\begin{array}{l}\text { Todas las localidades como } \\
\text { un solo grupo }\end{array}$ & 6,6 & 93,41 & $\mathrm{~N} / \mathrm{A}$ & $<0,001$ & 0,065 \\
\hline & $\begin{array}{l}\text { Un solo grupo sin tributarios } \\
\text { (río Saldaña) }\end{array}$ & 3,2 & 96,81 & $\mathrm{~N} / \mathrm{A}$ & $<0,001$ & 0,031 \\
\hline & $\begin{array}{l}\text { Cauce principal y represa } \\
\text { Betania como dos grupos }\end{array}$ & 1,7 & 98,23 & $\mathrm{~N} / \mathrm{A}$ & $<0,001$ & 0,017 \\
\hline \multirow{3}{*}{ STRUCTURE } & Entre poblaciones & $\mathrm{N} / \mathrm{A}$ & $\mathrm{N} / \mathrm{A}$ & 8,86 & $\mathrm{~N} / \mathrm{A}$ & $\mathrm{N} / \mathrm{A}$ \\
\hline & $\begin{array}{l}\text { Entre individuos dentro de } \\
\text { las poblaciones }\end{array}$ & $\mathrm{N} / \mathrm{A}$ & $\mathrm{N} / \mathrm{A}$ & 69,76 & $<0,001$ & 0,08 \\
\hline & Dentro de los individuos & $\mathrm{N} / \mathrm{A}$ & $\mathrm{N} / \mathrm{A}$ & 21,73 & $\mathrm{~N} / \mathrm{A}$ & $\mathrm{N} / \mathrm{A}$ \\
\hline
\end{tabular}




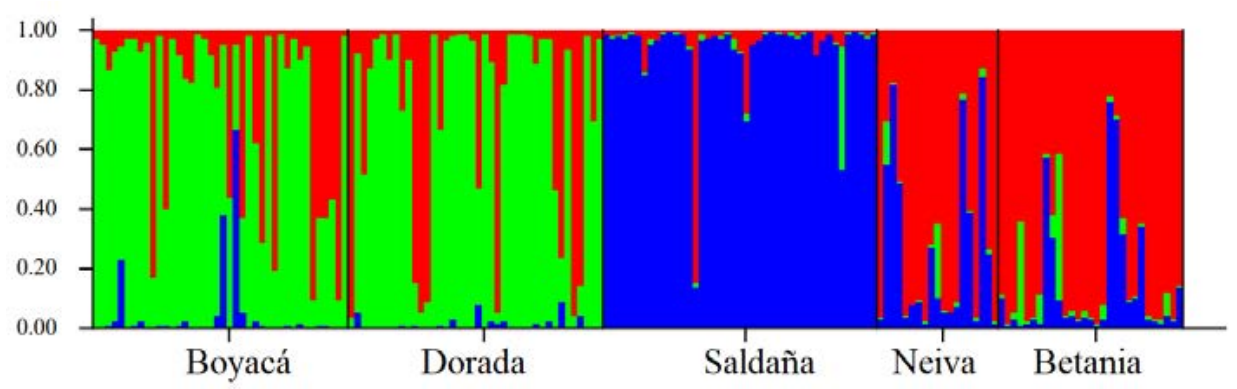

Figura 3. Análisis bayesiano de las poblaciones de Prochilodus magdalenae en las cinco localidades analizadas.

La mayor parte de los individuos de las localidades de Boyacá y La Dorada se asignaron al primer clúster (verde), mientras que en el caso de Neiva y Betania, la mayoría de los individuos se asignaron al segundo clúster (rojo). Finalmente, se identificó a la mayoría de los individuos presentes en el río Saldaña como una población asignada al tercer clúster (azul).

Se realizó un AMOVA, teniendo en cuenta la agrupación de los individuos por clúster independiente de la localidad de origen, para corroborar los resultados del análisis bayesiano obtenido en el programa STRUCTURE. Los resultados mostrados en la tabla 4 indican una moderada diferenciación para el estadístico Fst de 0,08 con un alto nivel de significancia.

\section{Discusión}

\section{Diversidad genética}

La creciente demanda sobre el recurso energético en Colombia ha generado la necesidad de fortalecer el campo hidroeléctrico con la construcción de represas que afectan la ictiofauna del río Magdalena. En Colombia se han documentado factores como el cambio en la oferta de alimento, aumento de especies invasoras o reducción de poblaciones con algún interés pesquero, por efecto de la construcción de represas (Jiménez-Segura et al., 2014). Mojica et al. (2012) mostraron la evolución histórica de las capturas totales de $P$. magdalenae en el río Magdalena, donde se observa la disminución gradual que han venido sufriendo las poblaciones de la especie; coincidiendo además con la construcción de la represa de Betania en 1987. No obstante, no se descartan otros factores antropogénicos más visibles sobre el recurso pesquero como lo es la sobrepesca o contaminación del 94 agua (Jiménez-Segura et al., 2014; Martínez-Silva, 2015).
Estos efectos no sólo son observables a nivel ecosistémicos (Agostinho et al., 1993); también, al igual que en otras especies, a nivel molecular se ha demostrado que el represamiento de los ríos influye en la baja diversidad genética de las especies. Tal es el caso reportado por Carvalho-Costa et al. (2008) y Rueda et al. (2011) para las especies $P$. costatus y $P$. lineatus en el río São Francisco (Brasil), donde registraron valores de diversidad por debajo de 0,5. Los datos presentados por estos autores muestran que entre los índices de He y Ho no exhiben una diferencia mayor a 0,2 en $P$. costatus y $P$. lineatus, mientras que los datos de Orozco y Narváez (2014) y Guevara (2015) para $P$. magdalenae en las cuenca del Magdalena y Catatumbo respectivamente, muestran una diferencia entre estos índices que supera los 0,5 , coincidiendo, de esta manera, con los datos obtenidos en el presente estudio (tabla 5).

En términos comparativos, no existen muchos trabajos que utilicen marcadores microsatélites para evaluar las características genéticas de las poblaciones de peces migratorios en Suramérica (Oliveira et al., 2009). Sin embargo, los valores de variabilidad genética registrados para P. magdalenae no son comparables a los observados para sus congéneres, así como también para otras especies de importancia comercial que realizan largas migraciones, ya que los niveles de heterocigosidad observada $(\mathrm{Ho})$ está entre los valores más bajos registrados para todo el grupo (tabla 5).

Cabe resaltar que en el estudio realizado por Orozco y Narváez (2014), se señala que existen localidades que se encuentran distantes de la represa de Betania, por ende no se tiene clara certeza que la baja variabilidad sea únicamente por causa de la fragmentación del ecosistema generada por esta represa. Sin embargo, no se descarta que los efectos localizados de represas como Hidrosogamoso u otras más pequeñas que se encuentran en el río Magdalena, estén influyendo en la baja variabilidad en 
esta población. Adicionalmente, es importante identificar si estas poblaciones han experimentado una reducción drástica en su tamaño, ya que esta reducción puede acelerar la pérdida de variabilidad genética y fijación moderada de alelos deletéreos, así como también reducir su potencial evolutivo al aumentar la probabilidad de extinción de la misma (Luikart y Cornuet, 1998; Vrijenhoek, 1998).

Fueron registrados desvíos en el HW para todos los loci analizados, y presentó un déficit significativo de heterocigotos, conforme a lo demostrado con las pruebas realizadas. Adicionalmente, este desvío se puede comprobar debido a los valores positivos registrados para el índice de fijación (F).

En estudios realizados con poblaciones de peces, es común encontrar este tipo de comportamiento (Carvalho-Costa et al., 2008; Hatanaka et al., 2006; Sanches, 2007; Silva, 2011). Estos autores, sugieren que la posible deficiencia de heterocigotos en una población puede ser generada por: errores de muestreo (subestimación de alelos), problemas técnicos como la presencia de alelos nulos, sttuttering o drop-out de alelos o efecto Whalund (exceso de homocigotos por solapamiento de poblaciones) y selección de alelos (García De León et al., 1997; Pereira et al., 2009, Xu et al., 2001).

El efecto Whalund implica la existencia de más de una población en cada una de las localidades muestreadas. Lo anterior se pudo corroborar en las poblaciones del bocachico con la ayuda de procedimientos bayesianos. Este resultado coincide con lo registrado por Orozco y Narváez (2014). Sin embargo, determinar específicamente cuál es la causa responsable del déficit de heterocigotos en una población no es una tarea fácil, pues se hace más complejo aún identificar cómo y cuáles son las interacciones de las poblaciones en medio naturales. Debido a lo anterior, el déficit de heterocigotos no puede ser explicado solo con una hipótesis, pues la interacción de los diferentes factores antes mencionados pueden estar contribuyendo a este resultado (Sanches, 2007).

Tabla 5. Análisis comparativo de los índices de variabilidad genética obtenido a partir marcadores moleculares microsatélites en algunas especies de peces migratorios presentes en ríos suramericanos. Donde Ra: riqueza alélica, Ho: variabilidad observada y He: variabilidad esperada.

\begin{tabular}{llrrrrl}
\hline Especie & Río & $\mathbf{N}^{\circ}$ Locus & Ra & Ho & He & Autor \\
\hline Prochilodus costatus & São Francisco & 6 & 7,6 & 0,45 & 0,66 & Carvalho-Costa et al., 2008 \\
Prochilodus costatus & São Francisco & 10 & 9,05 & 0,55 & 0,64 & Silva, 2011 \\
$\begin{array}{l}\text { Prochilodus argenteus } \\
\text { São Francisco }\end{array}$ & 4 & & $0,05-0,647$ & $0,05-0,91$ & Hatanaka et al., 2006 \\
Prochilodus argenteus & São Francisco & 8 & & 0,56 & 0,6 & Galzerani, 2007 \\
Prochilodus costatus & São Francisco & & & & 0,78 & Barroca et al., 2012a \\
Prochilodus argenteus & São Francisco & & & & 0,81 & Barroca et al., 2012b \\
Brycon hilarii & Miranda & 7 & 8,1 & 0,57 & 0,67 & Sanches, 2007 \\
Pseudoplatystoma corruscans & São Francisco & 6 & & 0,6981 & 0,73 & Dantas, 2010 \\
Prochilodus magdalenae & Sinú & 7 & & 0,273 & 0,854 & Santacruz-Beltrán, 2003 \\
Prochilodus magdalenae & Magdalena & 7 & 11,5 & 0,276 & 0,878 & Orozco y Narváez, 2014 \\
Prochilodus magdalenae & Catatumbo & 7 & & 0,23 & 0,84 & Guevara, 2015 \\
Prochilodus magdalenae & Magdalena & 7 & 0,21 & 0,83 & Este estudio \\
\hline
\end{tabular}


Los resultados obtenidos pueden estar relacionados con el comportamiento migratorio de $P$. magdalenae durante el año (Atencio, 2001; Cortés, 2003; Jiménez-Segura et al., 2010; Mojica et al., 2012), debido a que factores intrínsecos a las poblaciones también pueden contribuir a dicha pérdida de variabilidad. Un ejemplo de ello, es la reducción en el tamaño de la población debido a la sobrepesca más susceptibles durante la fase de migración reproductiva (Jiménez-Segura et al., 2014). Lo anterior conlleva a la pérdida en la población de individuos portadores de variabilidad genética adaptativa (alelos) y disminuye su potencial evolutivo. Otro factor importante a considerar es el repoblamiento mal direccionado a partir de "stocks" provenientes de granjas piscícolas que posiblemente pueden incrementar la homocigosis en medio natural.

Este hecho fue evidenciado a través de procedimientos bayesianos en la represa de Betania, en donde se mostró una homogeneidad genética de la población allí establecida. Asímismo, se resalta la relación genética entre las localidades de Neiva y Betania, lo que indica un flujo genético entre las mismas a pesar de la existencia de la barrrera. Esto se relaciona principalmente con el transporte manual de individuos y los programas de repoblamiento que se realizan en la represa de Betania (Fundación Humedales, 2008). Lo anterior puede ser un factor determinante en la baja variabilidad reportada en estos sectores dado que se desconoce el manejo de los alevines y los reproductores de bocachico que se están liberando dentro de la represa (Guevara, 2015).

Otro factor no menos importante, es la fragmentación de hábitat debido al relleno de las ciénagas, las cuales son utilizadas como sitio de alimentación y crianza de especies, construcción de embalses y represas como el caso de Betania, la cual fragmenta el acervo genético de la población. En consecuencia, la fragmentación y/o obstrucción de las rutas de migración altera la estructura y diversidad genética de esta especie, y se refleja tanto en los valores de variabilidad obtenidos en el presente estudio, así como también en otros modelos biológicos que presentan el mismo patrón migratorio, cuyos cauces han sido sujetos a la construcción de represas (Carvalho-Costa et al., 2008; Rueda et al., 2011; Sanches et al., 2012; Orozco y Narváez, 2014; Guevara, 2015) (tabla 5).

Los microsatélites evaluados permitieron demostrar el estado de vulnerabilidad en que se encuentra la especie, dada la baja diversidad genética que exhibió su población. No se descarta la posibilidad de que esta baja diversidad sea resultado de la presencia de alelos nulos y otros 96 artefactos técnicos y no por una causa biológica. A pesar de eso, es importante resaltar que estas poblaciones pueden estar expuestas a que cualquier factor ambiental pueda propiciar su extinción local (Kopas y Puentes, 2009), ya que aguas arriba de la represa, las poblaciones de especies endémicas del Magdalena con patrón migratorio de larga distancia, conocidas comúnmente como La Dorada (Brycon moorei), el Bagre Rayado (Pseudoplatystoma magdaleniatum Buitrago-Suárez y Burr, 2007) y la Picuda (Salminus affinis Steindachner, 1880) han desaparecido en su totalidad (Rodríguez, 2015).

\section{Estructura genética y diferenciación poblacional}

Según Laikre et al. (2005), los valores de variación genética de diferentes poblaciones reflejan la variabilidad genética total de una especie. Esta variación es vital ya que permite la acción de los diferentes mecanismos adaptativos frente a los retos impuestos por el ambiente. Sin embargo, hay que resaltar que la distribución de la variación genética dentro de una población no es homogénea y se estructura en grupos de individuos similares genéticamente (Laikre et al., 2005). Lo anterior es de suma importancia durante la aplicación de métodos genéticos para el manejo pesquero ya que si una especie posee poblaciones diferenciadas, el manejo deberá ser ejercido de forma separada (Waples et al., 2008), pues se estaría frente a diferentes unidades de manejo.

Los valores observados en el índice Fst global mostraron que existe una moderada diferenciación genética en el área considerada $(F s t=0,065)$, destacando la diferenciación existente entre la represa de Betania y todas las localidades consideradas en los análisis. El resultado proporcionado por el AMOVA indicó que existe una estructuración en la población de $P$. magdalenae en diferentes escalas geográficas donde se distribuye esta especie. Esta diferenciación puede estar relacionada con varios factores entre los que se puede encontrar, principalmente, la fragmentación que genera la represa en la cuenca (Pelicice et al., 2014) y el efecto de los ríos tributarios de la cuenca del Magdalena como sitios alternos para llevar a cabo el evento reproductivo. Carvalho-Costa et al. (2008) evidencian, a través del uso de microsatélites, que no hay sub-estructuración en la población de $P$. costatus aguas abajo de la represa Tres Marías, ubicada en el río Sao Francisco en Brasil. Sin embargo, Barroca et al. (2012a) mostraron que hay una marcada sub-estructuración en la población de esta especie cuando se tienen en cuenta los individuos ubicados aguas arriba y abajo de la represa Gafanhoto, en el río Pará. Lo anterior demuestra que los peces que se encuentran aguas abajo de la represa, tienden a diferenciarse de los que se encuentran aguas arriba 
por falta de un flujo génico. Esto permite corroborar un efecto negativo de la construcción de represas sobre las especies de peces migratorios, por lo que se deben generar programas más efectivos que mitiguen estas problemáticas.

Es así como en el presente estudio se muestra que la represa Betania, ubicada en el río Magdalena, genera una estructuración de la población de $P$. magdalenae debido a la incapacidad de los individuos para superar esta barrera y a la falta de estrategias que permitan la movilización de los individuos a través de la estructura (Carvalho-Costa et al., 2008). Sin embargo, a pesar de lo anterior, los resultados aportados por el análisis bayesiano exhibieron similitud entre la represa de Betania y Neiva, haciendo parte de la misma población (clúster rojo; figura 3). Lo anterior puede ser explicado por dos diferentes hipótesis: (1) es probable que esta relación esté determinada por eventos reproductivos que ocurren aguas arriba de la represa de Betania (JiménezSegura et al., 2014) y eventualmente logren atravesar la represa evidenciado un flujo unidireccional de individuos (Pelicice et al., 2014); y (2) unos de los mecanismos utilizados para disminuir los impactos generados por las represas han sido los programas de repoblamiento de peces, donde son transportados especímenes de importancia pesquera aguas arriba de la represa (Fundación Humedales, 2008). Dentro de estos especímenes está el bocachico, proveniente de estaciones piscícolas cuyos reproductores son capturados en inmediaciones de la represa de Betania (aguas abajo de la represa) y liberados aguas arriba en la misma Carrillo-Ávila et al. (2014). El manejo inadecuado a los individuos que se incluyen en los programas de repoblamiento contribuye de esta manera a la homogenización del acervo génico de una población (Guevara, 2015). Debido a lo anterior, en estas localidades comparten mucha información genética que no está determinada por mecanismos migratorios naturales.

Adicionalmente, Orozco y Narváez (2014) y Guevara (2015) encontraron que existe una moderada diferenciación genética entre $P$. magdalenae y $P$. reticulatus (sinónimo de $P$. magdalenae) respectivamente. Esto sugiere la coexistencia de las poblaciones de bocachico en la cuenca del río Magdalena (Orozco y Narváez, 2014). Dicha coexistencia es mantenida por los dos picos reproductivos del bocachico, lo que sugiere que una gran parte de la población migra durante el primer pico reproductivo y en el segundo migra aquella que no lo hizo en el primero (Jiménez-Segura et al., 2010; Orozco y Narváez, 2014). Por tanto, Orozco y Narváez (2014) consideran que esta estructuración es mantenida por eventos de "ondas reproductivas", donde estos grupos genéticamente diferenciados desovan en los mismos lugares en tiempos diferentes (Jorgensen et al., 2005). Se cree que este patrón reproductivo es común en muchas otras especies dentro de las cuencas (López-Casas et al., 2016).

Es importante resaltar la homogeneidad genética existente en el río Saldaña, que resulta muy diferente de las otras localidades. Esto puede ser evidencia de la afinidad que pueden tener los individuos a regresar a sitios particulares de la cuenca y realizar así su proceso reproductivo. Esta tendencia ha sido reportada en otras especies tropicales que realizan largas migraciones como el bocachico (Pereira et al., 2009; Guevara, 2015). Este tipo de comportamiento se ha observado también en $P$. argenteus usando herramientas como la radio-telemetría (Godinho y Kynard, 2006), aunque este no es exclusivo de peces dulceacuícolas, ya que también se ha documentado en especies marinas (Keeney et al., 2005).

\section{Agradecimientos}

El presente trabajo se pudo desarrollar en el marco de los proyectos: "Programa de genética de conservación para el Bocachico en la cuenca media y baja del río Magdalena" (convenio número 137-09 Ecopetrol-Universidad del Magdalena) y "Evaluación de la ecología molecular de los Bocachicos (Prochilodus spp.) Asociado a los ríos que drenan al Caribe colombiano" (con código de Colciencias 1117-489-25459) del Grupo de Investigación de Ecología y Biodiversidad Aplicada de la Universidad del Magdalena. Adicionalmente, agradecemos a todas las personas que aportaron al desarrollo del presente trabajo, en especial a María Cárdenas, Angie Angarita y Eber Romero.

\section{Referencias}

Agostinho, A., Vazzoler, A., Gomes, L. y Okada, E. 1993. Estratificación espacial y comportamiento de Prochilodus scrofa en distintas fases del ciclo de vida, en la planicie de inundación del alto del río Paraná y embalse de Itaipu, Paraná, Brasil. Revista de Hydrobiologia Tropical 26(1): 79-90.

Agostinho, A., Gomes, L. y Zalewski, M. 2001. The importance of floodplains for the dynamics of fish communities of the upper river Paraná. Ecohydrology and Hydrobiology 1(1): 209-217.

Agostinho, A., Pelicice, F. y Gomes, L. 2008. Dams and the fish fauna of the Neotropical region: Impacts and management related to diversity and fisheries. Brazilian Journal of Biology 68(4): 1119-1132.

Atencio, V. 2001. Producción de alevinos de especies nativas. MVZ-Córdoba 6(1): 9-14. 
Barroca, T., Arantes, F., Magalhaes, B., Siqueira, F., Horta, C., Pena, I., Dergam, J. y Kalapothakis, E. 2012a. Genetic diversity and population structure of Prochilodus costatus and Prochilodus argenteus preceding dam construction in the Paraopeba River, São Francisco River Basin, Minas Gerais, Brazil. Open Journal Genetics 2: 121-130.

Barroca, T., Santos, G., Duarte, N. y Kalapothakis, E. 2012b. Evaluation of geneic diversity population structure in a comercially important freshwater fish Prochilodus costatus (Characiformes, Prochilodontidae) using complex hypervariable repeats. Genetics and Molecular Research 1: $1-12$.

Bustamante, R. y Grez, A. 1995. Consecuencias ecológicas de la fragmentación de Bosques Nativos. Ambiente y Desarrollo 11(2):58-63.

Carrillo-Ávila, M., Espinosa-León, L., Cruz-Flor, W. y PerdomoAguirre, Y. 2014. Caracterización genética de Ichthyoelephas longirostris de los ríos La Miel y Ranchería usando marcadores microsatélites. Orinoquía 18(2):1-10.

Carvalho-Costa, L., Hatanaka, T., y Galetti, P. 2008. Evidence of lack of population substructuring in the Brazilian freshwater fish Prochilodus costatus. Genetics and Molecular Biology 31(1): 377-380.

Cortés, G.A. 2003. Guía para el manejo, cría y conservación del Bocachico Prochilodus magdalenae Steindachner, 1879. Convenio Andrés Bello. Bogotá D. C.

Dantas, H. 2010. Avaliação da estrutura genética do surubim, Pseudoplatystoma corruscans (Actinopterigy, Siluriformes) como subsidio para o repovoamento do submédio São Francisco. Masters Thesis Universidade Federal Rural do Pernambuco, Recife, Brazil.

Earl, D. y VonHoldt, B. 2012. Structure Harvester: a website and program for visualizing STRUCTURE output and implementing the Evanno method. Consevation Genetics 4(2): 359-361.

Evanno, G., Regnaut, S. y Goudet, J. 2005. Detecting the number of cluster of individuals using the software Structure: A simulation study. Molecular Ecology 14: 2611-2620.

Fundación Humedales. 2008. Evaluación pesquera y repoblamientos en el embalse de Betania: Fundación Humedales. Informe final de contrato para EGEMSA S.A. Bogotá D.C.

Galzerani, F. 2007. Análise da variabilidade genética de 98
Francisco, região de Três Marias, através de marcadores microsatélites. Monography Genetics and Evolution, Universidade Federal de São Carlos, São Carlos, Brazil.

García De León, Chikh, F.L. y Bonhomme, F. 1997. Microsatellite polymorphism and population subdivision in natural populations of European sea bass Dicentrarchus labrax (Linneo 1758). Molecular Ecology 6:51-62.

Godinho, A. y Kynard, B. 2006. Migration and spawning of radio-tagged zulega Prochilodus argenteus in a dammed Brazilian River. Transactions of the American Fisheries Society 135: 811-824.

Goudet, J. 1995. FSTAT: A program to estimate and test gene diversities and fixation indices (version 2.9.3).

Guevara, L. 2015. Evaluación de la estructura genética de la población silvestre y cultivada del Bocachico Prochilodus reticulatus (Characiformes: Prochilodontidae) asociada a la cuenca del río Catatumbo y a centros piscícolas en el departamento del Norte de Santander. Trabajo de Grado, Universidad Francisco de Paula Santander, Ocaña.

Guo, S. y Thompson, E. 1992. Performing the exact test of Hardy-Weinberg proportion for multiple aleles. Biometrics 48: 361-372.

Hatanaka, T., Silva, F. y Galetti, P. 2006. Population substructuring in a migratory freshwater fish Prochilodus argenteus (Characiformes, Prochilodontidae) from the São Francisco River. Genetica 126:153-159.

Hubisz, M., Falush, D., Stephens, M. y Pritchard, J. 2009. Inferring weak population structure with the assistance of sample group information. Molecular Ecology Resource 9: 1322-1332.

IDEAM - Cormagdalena. 2001. Estudio ambiental de la Cuenca Magdalena-Cauca y elementos para su ordenamiento territorial. Informe técnico. Acuerdo IDEAM - Cormagdalena convenio 003 de 1999. Bogotá, D.C.

Instituto Alexander von Humboldt IAVH. 2002. Sistema de indicadores de seguimiento de la política de Biodiversidad. Unidad de Sistemas de Información Geográfica SIG. Bogotá.

Jiménez-Segura, L., Palacio, J. y Leite, R. 2010. River flooding and reproduction of migratory fish species in the Magdalena river basin, Colombia. Ecology of Freshwather Fish 19: 178-186.

Jiménez-Segura, L., Restrepo-Santamaría, D., López-Casas, S., Delgado, J., Valderrama, M., Álvarez, J. y Gómez, D. 2014. Ictiofauna y desarrollo del sector hidroeléctrico en la 
cuenca del río Magdalena-Cauca, Colombia. Biota Colombiana 15(2): $3-25$.

Jorgensen, H., Hansen, M., Bekkevold, D., Ruzzante, D. y Loeschcke, V. 2005. Marine landscapes and population genetic structure of herring (Clupeaharengus L.) in the Baltic Sea. Molecular Ecology 14: 3219-3234.

Keeney, D., Heupel, M., Hueter, R. y Heist, E. 2005. Microsatellite and mitochondrial DNA analyses of the genetic structure of blacktip shark (Carcharhinus limbatus) nurseries in the northwestern Atlantic, Gulf of Mexico, and Caribbean Sea. Molecular Ecology 14: 1911-1923.

Kopas, J. y Puentes, A. 2009. Grandes represas en América ¿Peor el remedio que la enfermedad? Informe Técnico. Asociación Interamericana para la Defensa del Medio Ambiente AIDA. Oakland.

Laikre, L., Palm, S. y Ryman, N. 2005. Genetic population structure of fishes: implication s for coastal zone management. Ambio 34: 111-119.

Lasso, C., Gutierrez, F., Morales-Betancourt, M., Agudelo, E., Ramírez-Gil, H. y Ajiaco-Martínez, E. 2011. II. Pesquerías continentales de Colombia: cuencas del Magdalena-Cauca, Sinú, Canalete, Atrato, Orioco, Amazonas y vertiente del Pacífico. Serie Editorial Recursos Hidrobiológicos y Pesqueros Continentales de Colombia, Instituto de Investigación de los recursos Biológicos Alexander Von Humboldt. Bogotá, D.C.

López-Casas, S., Jiménez-Segura, L., Agostinho, A. y Pérez, C. 2016. Potamodromous migrations in the Magdalena River basin: bimodal reproductive patterns in neotropical rivers. Journal of Fish Biology 1-15.

Luikart, G. y Cornuet, J. 1998. Empirical evaluation of a test for identifying recently bottlenecked populations from allele frequency data. Conservation Biology 12(1):228-237.

Maldonado-Ocampo, J., Ortega-Lara, A., Usma, J., Galvis, G., Villa-Navarro, F., Vásquez, L., Prada-Pedreros, S. y Ardila, C. 2005. Peces de los Andes de Colombia. Instituto de Investigación de Recursos BiológicosAlexander Von Humboldt. Bogotá D.C.

Martínez-Silva, P. 2015. Variación espacio-temporal de microalgas acuáticas del embalse de Betania, Huila y su relación con la calidad del agua. Intropica 10: 11-19.

Mojica, J., Usma, J., Álvarez-León, R. y Lasso, C. 2012. Libro rojo de peces dulceacuícolas de Colombia. Instituto de Investigación de Recursos Biológicos Alexander von Humboldt, Instituto de Ciencias Naturales de la Universidad
Nacional de Colombia, WWF Colombia y Universidad de Manizales. Bogotá, D.C.

O'connell, M. y Wright, J. 1997. Microsatellite DNA in fishes. Reviews in Fish Biology and Fisheries. 7(1): 331-363.

Oliveira, C., Foresti, F. and Hilsdorf, A. 2009. Genetics of neotropical fish: From chromosomes to populations. Fish Physiology Biochemistry 35:81-100.

Orozco, G. y Narváez, J. 2014. Genetic diversity and population structure of Bocachico Prochilodus magdalenae (Pisces, Prochilodontidae) in the Magdalena River basin and its tributaries, Colombia. Genetics and Molecular Biology 37 (1): $37-45$.

Park, S., 2001. Trypanotolerance in Western African cattle and the population genetic effects of selection. Ph.D. Genetic Department, University of Dublin, Ireland.

Peakall, R. y Smouse, P. 2006. GenAlex6: Genetic analysis in Excel. Population genetic software for teaching and research. Molecular Ecology Resources (6): 288-295.

Pelicice, F., Pompeu, P. y Agostinho, A. 2014. Large reservoirs as ecological barriers to downstream movements of Neotropical migratory fish. Fish and Fisheries 1-19.

Pereira, L., Foresti, F. y Oliveira, C. 2009. Genetic structure of the migratory catfish Pseudoplatistoma corruscans (Siluriformes: Pimelodidae) suggest homing behaviour. Ecology of Freshwater Fish 18: 215-225.

Pompeu, P., Mascarenhas, L. y Barreira, C. 2009. Evaluation of the effects of pressure gradients on four brazilianfreshwater fish species. Brazilian Archives of Biology and Technology 52 (1): 111-118.

Ramírez, A. y Pinilla, G. 2012. Hábitos alimentarios, morfometría y estados gonadales de cinco especies de peces en diferentes períodos cilmáticos en el río Sogamoso (Santander, Colombia). Acta Biológica Colombiana 17 (2): 241-258.

Raymond, M. y Rousset, F. 1995. Testing Heterozygote excess and deficiency. Genetics 140: 1413-1419.

Rodríguez, M. 2015. ¿Para dónde va el río Magdalena? Riesgos sociales, ambientales y económicos del proyecto de navegabilidad. Friedich-Ebert-Stifungen Colombia (Fescol). Bogotá D.C.

Rueda, E., Sommer, J., Scarabotti, P., Markariani, R., y Ortí, G. 2011. Isolation and characterization of polymorphic 
microsatellite loci in the migratory freshwater fish Prochilodus lineatus (Characiformes: Prochilodontidae). Conservation Genetics Resource 3 (4): 681-684.

Sanches, A. 2007. Estrutura genética populacional de Brycon hilarii na sub-bacia do Rio Miranda, e seu significado para programas de conservação. Doctoral Thesis, UFSCar, São Carlos, Brazil.

Sanches, A., Galetti, P., Galzerani, F., Derazo, J., CutilakBianchi, B. y Hatakana, T. 2012. Genetic population structure of two migratory freshwater fish species (Brycon orthotaenia and Prochilodus argenteus) from the Sao Francisco River in Brazil and its significance for conservation. Latin American Journal of Aquatic Research 40 (1): 177-186.

Santacruz-Beltrán, D. 2003. Evaluación de la variabilidad genética conmarcadores microsatelites del Bocachico Prochilodus magdalenae (Steindachner 1878) en el Río Sinú, Colombia. Tesis de Grado, Universidad Nacional de Colombia, Bogotá.

Schneider, S., Roessli, D. y Excoffier, L. 2000. Arlequin: A software for population genetics data analysis. V.2.000. Genetics and Biometry Laboratory, Dept. of Anthropology, University of Geneva, Switzerland.

Silva, A. 2011. Estrutura genética populacional de Prochilodus costatus Valenciennes 1850 (Characiformes, Prochilodontidae) no alto São Francisco. Masters Thesis, Universidade Federal de São Carlos. São Carlos, Brazil.

Van Oosterhout, C., Hutchinson, W., Wills, D. y Shipley, P. 2004. MICRO-CHECKER: Software for identifyng and correcting genotyping errors in microsatellite data. Molecular Ecology Notes 4: 535-538.

Vrijenhoek, R. 1998. Conservation genetics of freshwater fish. Journal of Fish Biology. 53: 394-412.

Waples, R., Zabel, R., Scheuerell, M. y Sanderson, B. 2008. Evolutionary responses by native species to major anthropogenic changes to their ecosystems: Pacific salmon in the Columbia River hydropower system. Molecular Ecology 17:84-96.

Weir, B., y Cockerham, C. 1984. Estimating F-statistics for the analysis of population structure. Evolution 38 (6): 1358-1370.

Wright, E. 1978. Evolution and Genetics of Population. Vol. 2: The theory of gene frequencies. Press, London: University of Chicago.

Xu, Y., Liu, Y., Ridgway, N. y McMaster, C. 2001. Novel members of the human oxysterol-binding protein family bind phospholipids and regulate vesicle transport. The Journal of Biological Chemistry 276 (21):18407-18414.

Citar como: Fontalvo, P.P., Orozco-Berdugo, G. y Narváez-Barandica, J.C. 2018. Diversidad y estructura genética del Prochilodus magdalenae (Pisces: Prochilodontidae) aguas arriba y abajo de la represa Betania, Colombia. Intropica 13(2): 87-100. DOI: http://dx.doi.org/ 10.21676/23897864.2505. 